\title{
NGC 4654: polarized radio continuum emission as a diagnostic tool for a galaxy-cluster interaction
}

\section{Models versus observations}

\author{
M. Soida ${ }^{1}$, K. Otmianowska-Mazur ${ }^{1}$, K. Chyży ${ }^{1}$, and B. Vollmer ${ }^{2}$ \\ 1 Astronomical Observatory, Jagiellonian University, Orla 171, 30-244 Kraków, Poland \\ 2 CDS, Observatoire Astronomique de Strasbourg, UMR 7550, 11 rue de l'université, 67000 Strasbourg, France
}

Received 30 December 2005 / Accepted 30 June 2006

ABSTRACT

\begin{abstract}
Context. A recent comparison between deep VLA HI observations and dynamical models of the Virgo cluster spiral galaxy NGC 4654 has shown that only a model involving a combination of a tidal interaction and ram pressure can reproduce the data.

Aims. Deep radio polarization studies, together with detailed MHD modeling, can independently verify those conclusions, that are based on HI observations and dynamical models.

Methods. We performed deep polarized radio-continuum observations of the Virgo cluster spiral galaxy NGC 4654 with the Effelsberg $100 \mathrm{~m}$ telescope at $8.35 \mathrm{GHz}$ and the VLA at $4.85 \mathrm{GHz}$. Detailed 3D MHD simulations were made to determine the large-scale magnetic field and the emission distribution of the polarized radio continuum in the model, during the galaxy evolution within the cluster environment.

Results. This direct comparison between the observed and simulated polarized radio continuum emission corroborates the earlier results, that the galaxy had a recent rapid close encounter with NGC 4639 and is undergoing weak ram pressure by the intracluster medium.

Conclusions. This combination of deep radio polarization studies and detailed MHD modeling thus gives us unique insight into the interactions of a galaxy with its cluster environment. It represents a diagnostic tool that is complementary to deep HI observations.
\end{abstract}

Key words. galaxies: magnetic fields - galaxies: individual: NGC 4654 - galaxies: intergalactic medium - methods: numerical

\section{Introduction}

The bright Virgo cluster spiral galaxy NGC 4654 is located at the periphery of the cluster at a projected distance of $3.4^{\circ}=1 \mathrm{Mpc}^{1}$ from the cluster center (M 87). Its two-armed spiral structure is asymmetric with a shorter spiral arm to the northwest and a larger wider spiral arm to the southeast. Its HI distribution shows a sharp edge to the northwest and a large, very low column density tail to the southeast (Phookun \& Mundy 1995). While the northwestern part of the HI velocity field corresponds to a rising rotation curve, rotation is almost absent in the very low columndensity tail. The $\mathrm{H} \alpha$ image (Koopmann et al. 2001) shows enhanced star formation at the northwestern edge of the galactic disk.

Another spiral galaxy, NGC 4639, is located only $18^{\prime}=$ $90 \mathrm{kpc}$ in projection from NGC 4654 and might be considered to be a companion galaxy of NGC 4654. NGC 4639 has a ring-like HI distribution. Since it is located at the edge of the field observed by Phookun \& Mundy (1995) and Cayatte et al. (1994), it is not clear if its gas distribution and kinematics are perturbed. While Warmels (1988) observed extended gas in the east of NGC 4639, Cayatte et al. (1994) found atomic gas to the southwest.

Two kinds of interactions can in principle be responsible for the observed asymmetries and perturbations of NGC 4654: (i) a gravitational interaction with a massive galaxy or (ii) ram pressure due to the galaxy's rapid motion within the hot and tenuous

\footnotetext{
${ }^{1}$ We use a distance of $17 \mathrm{Mpc}$ to the Virgo cluster.
}

intracluster medium. In order to investigate the recent evolutionary history of NGC 4654, Vollmer (2003) made numerical simulations including a gravitational interaction, ram pressure, and a combination of both, concluding that

- a past ram-pressure stripping event where the galaxy has already passed the cluster center $800 \mathrm{Myr}$ ago and is now emerging from the cluster core gives rise to a low surfacedensity tail in the southeast of the galaxy center. This tail shows clear signs of rotation;

- a gravitational interaction between NGC 4654 and NGC 4639 cannot produce a low surface density tail;

- only a gravitational together with a relatively small amount of ram pressure leads to the observed low surface-density tail where rotation is almost absent.

The results of Vollmer (2003) suggest that NGC 4654 had a tidal interaction with another massive galaxy in the recent past ( $~ 500 \mathrm{Myr}$ ago). A model where the position and radial velocity of the perturbing massive galaxy are reasonably close to those of NGC 4639 can explain the perturbed stellar distribution and the gas distribution of the inner disk. Therefore, Vollmer (2003) identified NGC 4639 as the perturbing galaxy.

A caveat of the gravitational interaction scenario is that NIR Tully-Fisher measurements yield a line-of-sight distance between NGC 4654 and NGC 4639 of more than 5 Mpc (Gavazzi et al. 1999). While NGC 4654 is located 1-2 Mpc in front of the cluster core, NGC 4639 is located far behind $(\sim 5 \pm 1 \mathrm{Mpc})$. Observations of Cepheids give independent distance estimates 
for a number of Virgo cluster galaxies (Gibson et al. 2000; Freedman et al. 2001). Five galaxies are in both, the Gavazzi et al. (1999) and the Freedman et al. (2001) samples. For 4 galaxies (NGC 4321, NGC 4535, NGC 4536, and NGC 4536), the difference between the distance moduli obtained from the two methods are small $\left(\left|\Delta \mu_{0}\right|<0.2\right)$. However, this difference is much larger for NGC $4639\left(\left|\Delta \mu_{0}\right|=0.4\right)$. The Cepheid distance estimates place NGC 4639 closer, but still a few Mpc behind the center of the Virgo cluster (M 87). Thus these measurements corroborate the large NIR Tully Fisher distance of NGC 4639.

Since the preferred scenario of Vollmer et al. (2003) includes ram pressure of $p_{\text {ram }}=200 \mathrm{~cm}^{-3}\left(\mathrm{~km} \mathrm{~s}^{-1}\right)^{-1}$, a galaxy velocity of $\sim 1000 \mathrm{~km} \mathrm{~s}^{-1}$ implies an intracluster medium (ICM) density of $n_{\mathrm{ICM}} \sim 10^{-4} \mathrm{~cm}^{-3}$. At the distance of NGC 4639, the ICM density according to Schindler et al. (1999) is lower than $10^{-5} \mathrm{~cm}^{-3}$. This suggests that either (i) NGC 4654 had a gravitational interaction with another massive object; (ii) the Tully Fisher method underestimates the line-of-sight position of NGC 4654, (i.e. NGC 4654 is too bright with respect to its rotation velocity); or (iii) the ICM distribution is elongated along the line-of-sight, similar to the galaxy distribution (Gavazzi et al. 1999). In the case of scenario (i) the perturber has to be a massive dark galaxy (see, e.g., Minchin et al. 2005) since there is no other visible companion than NGC 4639.

In this article we test the viability of the preferred scenario of Vollmer (2003). We used polarized radio-continuum observations of NGC 4654, together with an MHD model, to put further constraints on the models. The radio continuum emission is due to relativistic electrons that gyrate around the galactic magnetic field (synchrotron emission). If the polarized radiocontinuum emission (called PRCE hereafter) is free of Faraday rotation (typically at frequencies above $\sim 4 \mathrm{GHz}$ ), it traces the ordered large-scale magnetic field directly. This magnetic field is extremely sensitive to shear and compression motions, which are not easily detected in 2D velocity fields. Using polarized radiocontinuum emission at $6 \mathrm{~cm}$, Vollmer et al. (2004) could unambiguously identify the ram-pressure compressed region in the Virgo cluster spiral galaxy NGC 4522. We were able to reproduce the HI distribution, velocity field, and the distribution of polarized radio-continuum emission with our model simulations (Vollmer et al. 2006).

In order to understand the behavior of the large-scale magnetic field and thus the polarized radio-continuum emission during a ram-pressure stripping event, Otmianowska-Mazur \& Vollmer (2003) solved the induction equation on the velocity fields provided by a dynamical model. They showed that a prominent maximum of polarized radio emission is observed during compression and in a state where the galaxy has passed the cluster center, the ram-pressure decreases, and the gas pushed by ram pressure falls back onto the galactic disk. We applied the method described in Otmianowska-Mazur \& Vollmer (2003) to NGC 4654; i.e., we solve the induction equation on the velocity fields of the simulations of Vollmer (2003) and compare the results to our polarized radio-continuum observations.

The Effelsberg and VLA radio continuum observations are presented in Sect. 2. In Sect. 3 we explain the 3D MHD model and how we calculated the model of polarized radio-continuum emission maps. The results of our model calculations are shown in Sect. 4 followed by direct comparison between our observations and simulations (Sect. 5). These results are discussed in Sect. 6 and we give our conclusions in Sect. 7 .

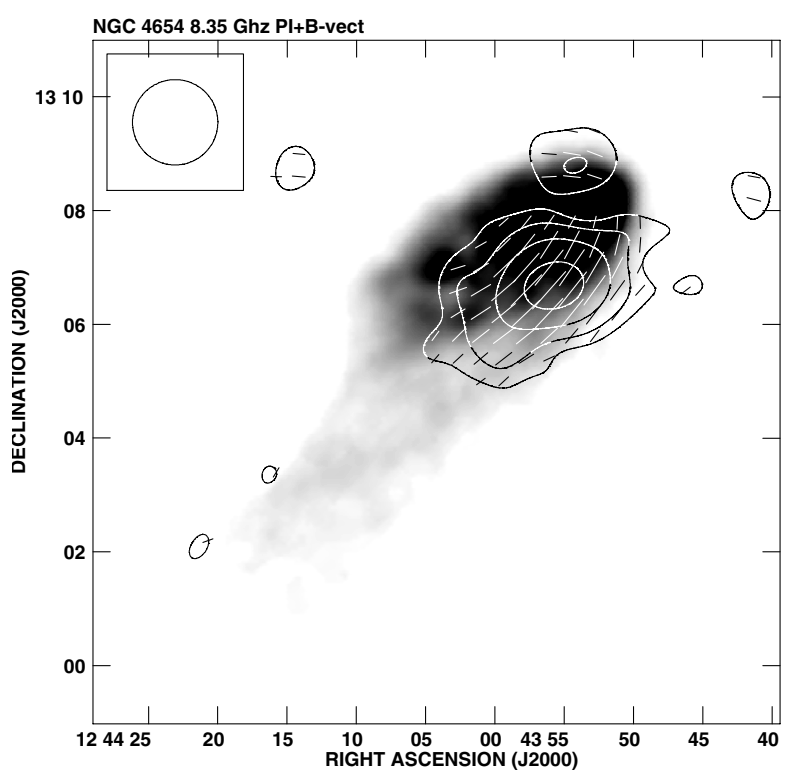

Fig. 1. Contours of polarized intensity of NGC 4654 at $8.35 \mathrm{GHz}$ with magnetic $\boldsymbol{B}$-vectors proportional to the polarized intensity superimposed onto the HI emission. The contour levels are $(3,5,8,12) \times$ $0.039 \mathrm{mJy} / \mathrm{b}$.a. The map resolution is $90^{\prime \prime}$. Greyscale: HI gas distribution (Phookun \& Mundy 1995).

\section{Radio observations of NGC 4654}

Radio polarimetric observations of NGC 4654 were performed at $8.35 \mathrm{GHz}$ using the single-horn receiver in the secondary focus of the 100-m Effelsberg radio telescope ${ }^{2}$. The galaxy has been scanned alternatively in RA and Dec. Four data channels were recorded: two total power channels (then averaged to a single total power Stokes $I$ channel) and two correlation channels containing the Stokes $Q$ and $U$ parameters. We obtained 33 coverages.

The telescope pointing was checked at time intervals of about $1.5 \mathrm{~h}$ by making cross-scans of a nearby strong point source. The flux-density scale was calibrated by mapping the polarized source 3C 138 and computing its total power flux densities of $2.49 \mathrm{Jy}$ at $8.35 \mathrm{GHz}$ using the formulae of Baars et al. (1977). The data reduction was performed using the NOD2 data reduction package (Haslam 1974). The coverages were combined into final $I, U$, and $Q$ maps using the spatial frequency weighting method (Emerson \& Gräve 1988).

The distributions of Stokes parameters were combined into final maps of total and polarized intensity, polarization degree, and polarization position angle. Noisy structures smaller than the telescope beam-width were removed using a digital filtering. A convolution to the beam-width of $90^{\prime \prime}$ was applied to increase the sensitivity to the extended polarized emission. The rms noise level in the final map of polarized intensity at $8.35 \mathrm{GHz}$ is $0.039 \mathrm{mJy} / \mathrm{b} . \mathrm{a}$.

NGC 4654 was observed, together with other large spirals of the Virgo Cluster, and a detailed analysis of their radio total and polarimetric properties will be discussed elsewhere. We also observed those galaxies at $4.85 \mathrm{GHz}$ with a resolution of $2.5^{\prime}$. The contour map of polarized intensity of NGC 4654 at $8.35 \mathrm{GHz}$ is presented in Fig. 1 with superimposed $\boldsymbol{B}$-vectors of polarized intensity (i.e. $\boldsymbol{E}$-vectors rotated by $90^{\circ}$ ), overlaid upon the

\footnotetext{
2 The 100-m telescope at Effelsberg is operated by the Max-PlanckInstitut für Radioastronomie (MPIfR) on behalf of the Max-PlanckGesellschaft.
} 


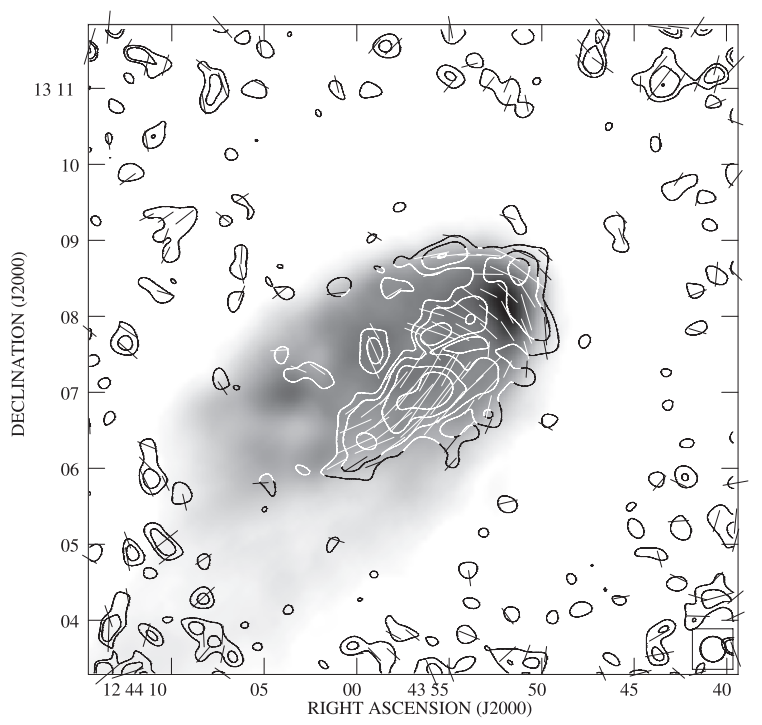

Fig. 2. Contours of polarized intensity of NGC 4654 at $4.85 \mathrm{GHz}$ with magnetic $\boldsymbol{B}$-vectors proportional to the polarized intensity superimposed onto the $\mathrm{HI}$ emission. The contour levels are $(3,5,8,12,20)$ $\times 10 \mu \mathrm{Jy} / \mathrm{b}$.a. The map resolution is $30^{\prime \prime}$. Greyscale: HI gas distribution (Phookun \& Mundy 1995).

HI emission. The polarized emission shows strong North-South asymmetry that may impose a compression or stretching of magnetic fields on the southern side of the galaxy. Similar polarized structure is also visible in our lower-resolution $4.85 \mathrm{GHz}$ observations.

The VLA observations represent part of a larger survey of 8 Virgo spiral galaxies that were observed in polarized radio continuum emission at 6 and $20 \mathrm{~cm}$ (PI: B. Vollmer). Since allof the data will be presented in a forthcoming article, we only show the polarized radio continuum emission at $6 \mathrm{~cm}$.

NGC 4654 was observed at $4.85 \mathrm{GHz}$ during $5 \mathrm{~h}$ on December 3 and 15, 2005 with the Very Large Array (VLA) of the National Radio Astronomy Observatory (NRAO) ${ }^{3}$ D array configuration. The band pass was $2 \times 50 \mathrm{MHz}$. We used 3C 286 as the flux calibrator and $1254+116$ as phase calibrator, which was observed every $30 \mathrm{~min}$. Maps of $17^{\prime \prime}$ resolution were made using the AIPS task IMAGR with ROBUST $=3$, which is close to pure natural weighting (5). The maps were convolved to a beam size of the final maps of $20^{\prime \prime} \times 20^{\prime \prime}$. We ended up with an rms of $10 \mu \mathrm{Jy} /$ beam in total and polarized emission. In Fig. 2 the contour map of polarized intensity of NGC 4654 at $4.85 \mathrm{GHz}$ is presented, together with superimposed $\boldsymbol{B}$-vectors of polarized intensity (i.e. $\boldsymbol{E}$-vectors rotated by $90^{\circ}$ ), overlaid upon the HI emission.

Similar to the Effelsberg data, the VLA data show a prominent ridge of polarized emission in the south of the galaxy. Moreover, this ridge extends to the southwest. The VLA data show that the lack of polarized emission in the Effelsberg data at the tip of the HI head is due to strong beam depolarization since the magnetic field changes direction strongly following the HI and optical envelopes (Fig. 2). The magnetic vectors encircle the HI head-like structure in the northwestern part of the galaxy.

When we convolved the VLA data to the Effelsberg resolution, we found two major differences between the VLA and Effelsberg maps: (i) the northern maximum is more extended in the convolved VLA map, which means that the Effelsberg

${ }^{3}$ NRAO is a facility of National Science Foundation operated under cooperative agreement by Associated Universities, Inc. telescope did not detect all the flux there (the northern maximum is smaller than the Effelsberg beam; cf. Fig. 1); and (ii) the southwestern part of the main ridge of polarized emission in the Effelsberg data seems to extend into the extended HI tail. Since this is not observed in the VLA data, this additional emission in the Effelsberg data is most probably due to the large beam.

Both features: the asymmetry in the polarized emission and the geometry of the magnetic field in NGC 4654 should be explained by our MHD modeling and can constitute a powerful tool for distinguishing between numerical experiments and thus between different scenarios of gas dynamics (Vollmer 2003) and the magnetic field evolution in this galaxy.

\section{Model}

The 3D MHD model of magnetic-field evolution (see Otmianowska-Mazur \& Vollmer 2003, for more details) is applied to three selected numerical experiments of the Virgo galaxy NGC 4654 presented in Vollmer (2003). We take only those cases into consideration where stars are treated as a noncollisional component (Vollmer 2003):

Model GR: the gravitational (tidal) interaction with the second galaxy;

Model RPS: the ICM ram pressure;

Model GRPS: the gravitational interaction and constant ICM ram pressure.

The Zeus3D MHD code (Stone \& Norman 1992a,b) is used to solve the induction equation

$\frac{\partial \boldsymbol{B}}{\partial t}=\nabla \times(\boldsymbol{v} \times \boldsymbol{B})-\nabla \times(\eta \nabla \times \boldsymbol{B})$

where $\boldsymbol{B}$ is the magnetic induction, $\boldsymbol{v}$ the large-scale velocity of the gas, and $\eta$ the coefficient of a magnetic diffusion (see Otmianowska-Mazur \& Vollmer 2003). Realistic, timedependent gas-velocity fields are provided by the 3D $N$-body sticky-particle computations of HI cloud complexes evolving in a galactic disk, a bulge and a halo simulated by non-collisional particles (see Vollmer 2003; and Otmianowska-Mazur \& Vollmer 2003). The clouds collide inelastically, and in the two experiments, they are affected by the ICM ram pressure due to motion of the galaxy with respect to the ICM.

The velocity obtained from the $N$-body code has a discrete distribution. In order to solve the induction equation (Eq. (1)) with the help of Zeus3D code, we have to interpolate these velocities to the regular grid. In Otmianowska-Mazur \& Vollmer (2003) we applied a spline function with a density-dependent smoothing length. It turned out that we had to use a large smoothing length to suppress the noise in the velocity field of the outer disk, which was due to a small, local particle density. In this way we avoided numerical artifacts of the magnetic field distribution in the outer disk layers, but at the same time we obtained a magnetic field distribution beyond the edges of the gas distribution. For these reasons we decided to apply another method to solve the interpolation problem. The method is based on the kriging interpolation (see e.g. Isaaks \& Srivastava 1989) modified to be used effectively in our $3 \mathrm{D}$ case.

\subsection{Kriging interpolation and our modification}

Kriging is a family of linear least-square estimation algorithms. It is used to approximate or interpolate data. The end result of kriging is to obtain the conditional expectation as a best estimate 
for all unsampled locations in a field and, consequently, a minimized error variance at each location. The kriging interpolation assumes (see e.g. Isaaks \& Srivastava 1989) that the value of any (vector) field at a point $y(x)$ can be approximated by a weighted linear combination of a discrete set of vectors given at a certain set of points $y_{i}=y\left(x_{i}\right)$ (provided by the $N$-body simulations, in our case)

$y(x)=\sum_{i=1}^{N} y_{i} w_{i}(x)$

where the weight coefficients $w_{i}(x)$ determine to what extent $y_{i}$ influences $y(x)$. The best approximation condition requires that the weights' sum has to be normalized by $\sum_{i=1}^{N} w_{i}(x)=1$. They can be determined from the set of equations

$\sum_{i=1}^{N} d_{i j} w_{i}(x)+\lambda(x)=d_{j}(x)$

where $d_{i j}=d\left(x_{i}, x_{j}\right)$ and $d_{i}(x)=d\left(x_{i}, x\right)$ are called "covariance coefficients". These coefficients describe how closeby the points $x_{i}$ and $x_{j}$ (or $x_{i}$ and $x$ ) are distributed. In our 3D case, we chose $d$ falling exponentially with the distance $d_{i j}=\mathrm{e}^{-\left\|x_{i}-x_{j}\right\|}$ and $d_{i}(x)=\mathrm{e}^{-\left\|x_{i}-x\right\|}$. A slack variable (the Lagrange coefficient) $\lambda(x)$ is introduced here to balance numbers of unknowns with $N+1$ equations ( $N$ for each $w_{i}(x)$ plus the normalization equation). This method implies determining (to solve a set of $N+1$ equations) $w_{i}(x)$ and $\lambda(x)$ for each point of interpolation $x-$ in $171 \times 171 \times 71$ points in our case.

We noticed that we can substantially reduce the calculation time. If we define a new set of unknowns $g_{i}, i=0,1, \ldots, N-1$ and a new slack variable $\kappa$ fulfilling the set of equations

$$
\begin{aligned}
\sum_{i=0}^{N-1} d_{i j} g_{i}+\kappa & =y_{j} \\
\sum_{i=0}^{N-1} g_{i} & =0,
\end{aligned}
$$

then, multiplying equations (3) by $g_{j}$ and summing over $j$, we get

$\sum_{i=0}^{N-1} w_{i}(x) \sum_{j=0}^{N-1} d_{j i} g_{j}+\lambda(x) \sum_{j=0}^{N-1} g_{j}=\sum_{j=0}^{N-1} g_{j} d_{j}(x)$

where we make use of the symmetry of $d_{i j}$. The second term vanishes (Eq. (5)), and using Eq. (4), we can rewrite it as

$\sum_{i=0}^{N-1} w_{i}(x)\left(y_{i}-\kappa\right)=\sum_{j=0}^{N-1} g_{j} d_{j}(x)$.

Finally, by substituting Eq. (2) we obtain the following formula for the interpolated value

$y(x)=\sum_{i=0}^{N-1} g_{i} d_{i}(x)+\kappa$.

Thus, for all interpolation points we solve set of Eqs. (4) and (5) only once, and for each point $x$ we calculate values $d_{i}(x)$ and their scalar product with $g_{i}$.

We applied this interpolation routine only to the velocityfield components. For interpolation of the mass distribution (used only for figures), we used a much faster method - smoothing particle masses with the Gaussian function with smoothinglength small enough to see gaseous features.

\subsection{Model input parameters}

The dynamical models are fully described in Vollmer et al. (2003). As in our previous paper (Otmianowska-Mazur \& Vollmer 2003), the induction equation is solved in rectangular coordinates $(X Y Z)$. We use $171 \times 171 \times 71$ grid points along the $X, Y$ and $Z$ axis, respectively. The grid spacing is $400 \mathrm{pc}$ in all directions, which gives the size of our modeled box as $68.4 \mathrm{kpc} \times 68.4 \mathrm{kpc} \times 28.4 \mathrm{kpc}$.

We assume the magnetic field to be partially coupled to the gas via the turbulent diffusion process (Otmianowska-Mazur et al. 2000) assuming the magnetic diffusion coefficient to be $\eta=5 \times 10^{-25} \mathrm{~cm}^{2} / \mathrm{s}$. The initial magnetic field is purely toroidal with a strength of $10 \mu \mathrm{G}$ at a radius $2 \mathrm{kpc}$ and falling linearly to zero toward $R=0$. The magnetic-field strength is constant between $2 \mathrm{kpc}<R<20 \mathrm{kpc}$ and decreases with a Gaussian profile for $20 \mathrm{kpc}<R<30 \mathrm{kpc}$ with a half width of $500 \mathrm{pc}$. It is zero for $R>30 \mathrm{kpc}$. In the vertical direction, the initial magnetic field strength is a Gaussian function with a half width of $500 \mathrm{pc}$.

\subsection{The polarization maps}

In order to obtain the simulated polarized intensity maps, we rotate the cube of $3 \mathrm{D}$ magnetic field according to the orientation of NGC 4654. Next, we integrate the transfer equations of synchrotron emissivities in Stokes $I, Q$, and $U$ parameters along the line of sight:

$\frac{\mathrm{d}}{\mathrm{d} l}\left(\begin{array}{c}I \\ Q \\ U\end{array}\right)=\left(\begin{array}{ccc}\epsilon_{I} & 0 & 0 \\ p \epsilon_{I} \cos 2 \chi & \cos \Delta & -\sin \Delta \\ p \epsilon_{I} \sin 2 \chi & \sin \Delta & \cos \Delta\end{array}\right)\left(\begin{array}{c}1 \\ Q \\ U\end{array}\right)$

where the synchrotron emissivity is

$\epsilon_{I} \propto n_{\mathrm{rel}} B_{\perp}^{(\gamma+1) / 2}$

We apply here the value of the relativistic electrons spectral in$\operatorname{dex} \gamma=2.8$ and the Faraday rotation angle

$\Delta \propto \lambda^{2} \int n_{\mathrm{th}} B_{\|} \mathrm{d} s$

The subscripts $\perp$ and $\|$ are used with respect to the line-of-sight, and $\chi$ is the position angle of the sky-projected magnetic-field vector. The integration in Eq. (11) is along the line of sight, and observations wavelength is $\lambda$.

The intrinsic degree of synchrotron polarization is assumed to be $p=75 \%$. Both thermal and relativistic electron distributions are taken as Gaussian

$n_{\text {rellth }} \propto \mathrm{e}^{-\left(R / R_{0}\right)^{2}} \mathrm{e}^{-\left(z / z_{0}\right)^{2}}$

where the radial scale-length $R_{0}$ is set to $10 \mathrm{kpc}$ and the scaleheight $z_{0}$ to $1 \mathrm{kpc}$. We neglect Faraday effects by setting $n_{\text {th }}=0$.

Finally for direct comparison with radio observations, the model maps of Stokes $I, Q$, and $U$ are convolved with a 2D Gaussian function with a $H P B W$ of $20^{\prime \prime}$ and $80^{\prime \prime}$. The final $Q$ and $U$ maps are combined to obtain maps of polarized intensity and polarization angle (rotated by $90^{\circ}$ to show the magnetic vector).

As described in Sect. 1 the polarized radio-continuum emission traces the ordered large-scale magnetic field. In general, the total magnetic field of a spiral galaxy can be divided into two parts (see e.g. Beck et al. 1996): (i) a small-scale (smaller than a few hundred pc) magnetic field that is connected to the turbulent motions of the interstellar medium and (ii) a large-scale (larger 
than a few hundred pc) ordered regular magnetic field that is most probably due to a large-scale $\alpha \Omega$-dynamo. The large-scale magnetic field can be amplified by shear or compression motions of the interstellar medium where the magnetic field is frozen in. The small-scale magnetic field is amplified by the turbulent motions of the interstellar medium (small-scale dynamo).

The polarized radio-continuum emission is sensitive to a combination of magnetic-field compression or amplification. In addition, it is sensitive to the average field ordering along the line of sight, which is a pure geometrical effect depending on the position and inclination angle of the galaxy. To separate these effects one can use the total power radio continuum map to calculate the degree of polarization, which is only sensitive to the degree of intrinsic field ordering.

As explained at the beginning of this section, we only model the large-scale magnetic field (Eq. (1)). We do not have access to the small-scale magnetic field, which would require a model of the turbulent interstellar medium together with a small-scale dynamo. Therefore, we are not able to calculate a total power map for the model. However, we plan for the future to tie the distribution of the relativistic electrons (Eq. (12)) to the $\mathrm{H} \alpha$ emission distribution. This emission traces ionizing $\mathrm{O}$ and $\mathrm{B}$ stars that will soon explode as supernovae giving rise to relativistic electrons. If we assume that the star formation history is approximately constant over the past ten million years, the $\mathrm{H} \alpha$ emission represents a reasonable measure for the relativistic electron distribution. We can try to link the total power map to the $\mathrm{H} \alpha$ emission using equipartition between the turbulent kinetic energy of the interstellar medium (which is connected to the star formation rate and thus to the $\mathrm{H} \alpha$ emission) and the total magnetic energy.

\section{Results}

For the moment, we use the polarized radio-continuum emission for the comparison between our models and observations. We show in the following that the information contained in the polarized radio-continuum is enough to distinguish between the different dynamical models.

\subsection{Gravitational interaction alone (model GR)}

Figure 3 shows the evolution of the polarized intensity distribution for the model of tidally interacting galaxies. The timestep of each snapshot is plotted in the upper left corner of each panel. The closest encounter between NGC 4654 and NGC 4639 occurs at $t=460 \mathrm{Myr}$. The images are convolved to a resolution of $20^{\prime \prime} \mathrm{HPBW}$. This figure can be directly compared to Fig. 12 of Vollmer (2003), because we present snapshots exactly at the same time steps. Figure 12 of Vollmer (2003) also shows the positions of the interacting galaxy NGC 4639. We use the inclination and position angle of NGC $4654\left(i=50^{\circ}, \mathrm{PA}=120^{\circ}\right)$.

The magnetic field distribution starts with an axisymmetric, purely azimuthal configuration. Since the polarized radiocontinuum emission is subject to Faraday rotation, i.e. the rotation of the magnetic field vector in the plane of the sky due to the magnetic field component along the line of sight, projection effects are important. The magnetic field component along the line of sight is largest at the two ends of the galaxy's major axis. Therefore, Faraday depolarization suppresses the polarized radio-continuum emission there. Since the polarized radio-continuum emission depends on the line-of-sight integration of the component of the magnetic field vector that lies in the plane of the sky, geometrical effects are important. And since this component of the magnetic-field vector is changing direction rapidly along the major axis, the corresponding polarized radio-continuum emission is suppressed there. As a result, the synchrotron emission distribution shows two maxima to the northeast and southwest of the major axis. The first snapshot in Fig. 3 shows the polarized radio-continuum emission calculated using a slightly evolved magnetic field ( $t=300 \mathrm{Myr})$. During the galaxy evolution, non-azimuthal gas flows induce radial components of the magnetic field resulting in the non-zero magnetic pitch angles without applying an explicit $\alpha$ dynamo mechanism. This effect becomes visible as a displacement of the polarized intensity maxima along the major axis (see e.g. Knapik et al. 2000).

At $t>460 \mathrm{Myr}$, the gravitational interaction with NGC 4639 perturbs the gas flow leading to regions of gas compression and/or shear. Due to electromagnetic induction (Eq. (1)), the magnetic field is enhanced in these regions. Thus, the polarized intensity maxima follow gas compression and shear regions, but because the timescale of turbulent diffusion is larger than the viscous timescale of the gas, there is no one-to-one correspondence between them. In some places and at some timesteps, gaseous and polarization features coincide, while in other places and at other timesteps, arms of polarized radio continuum emission lie just between gaseous ones (e.g. the northwestern maximum at $t=700 \mathrm{Myr}$ ). More detailed analysis of polarized intensity distribution shows that in general the maxima are left behind gas compression regions. This is not clearly visible in polarized intensity plots, as details are smoothed-out due to the line-of-sight integration and beam convolution.

Further evolution $(t>800 \mathrm{Myr})$ shows a weakening of the magnetic field and thus a weakening of the polarized radiocontinuum emission. In regions where gas falls back onto the galactic disk such as at the basis of the southern tidal tail at $t \geq 900 \mathrm{My}$, the magnetic field is efficiently amplified, and maxima of polarized emission reappear. In the final time step $(t=1000 \mathrm{Myr})$, two comparable maxima of polarized radio continuum emission coincide with gas compression or shear regions.

\subsection{Ram pressure alone (model RPS)}

The second simulation only includes a strong ram-pressure stripping event. The galaxy is diving deeply into the cluster potential where ram pressure becomes stronger due to the increased velocity of the galaxy and the higher intracluster medium density. The closest approach of the galaxy to the cluster center (M 87), and thus maximum ram pressure occurs at $t=0 \mathrm{Myr}$. The magnetic field evolution is strongly influenced by the gas compression and shear motions induced by ram pressure (Fig. 4). This figure can be directly compared to Fig. 3 presented in Vollmer (2003), because we present model snapshots at the same time steps. The direction of the wind is identical to Fig. 3 of Vollmer (2003). The inclination angle between the galactic and the orbital plane is $i=0^{\circ}$. The temporal ram pressure profile is a Lorentzian with a maximum ram pressure of $p_{\text {ram }}=5000 \mathrm{~cm}^{-3}\left(\mathrm{~km} \mathrm{~s}^{-1}\right)^{2}$. In Otmianowska-Mazur \& Vollmer (2003) we calculated the evolution of the distribution of polarized radio-continuum emission for a similar ram-pressure stripping event where the inclination angle between the galactic and the orbital plane was $i=20^{\circ}$ and maximum ram pressure was $p_{\text {ram }}=2000 \mathrm{~cm}^{-3}$ $\left(\mathrm{km} \mathrm{s}^{-1}\right)^{2}$. The evolution of the distribution of polarized radiocontinuum emission (Fig. 4) shows the same characteristics as that of Otmianowska-Mazur \& Vollmer (2003): due to the strength of the interaction, the magnetic field distribution and, 

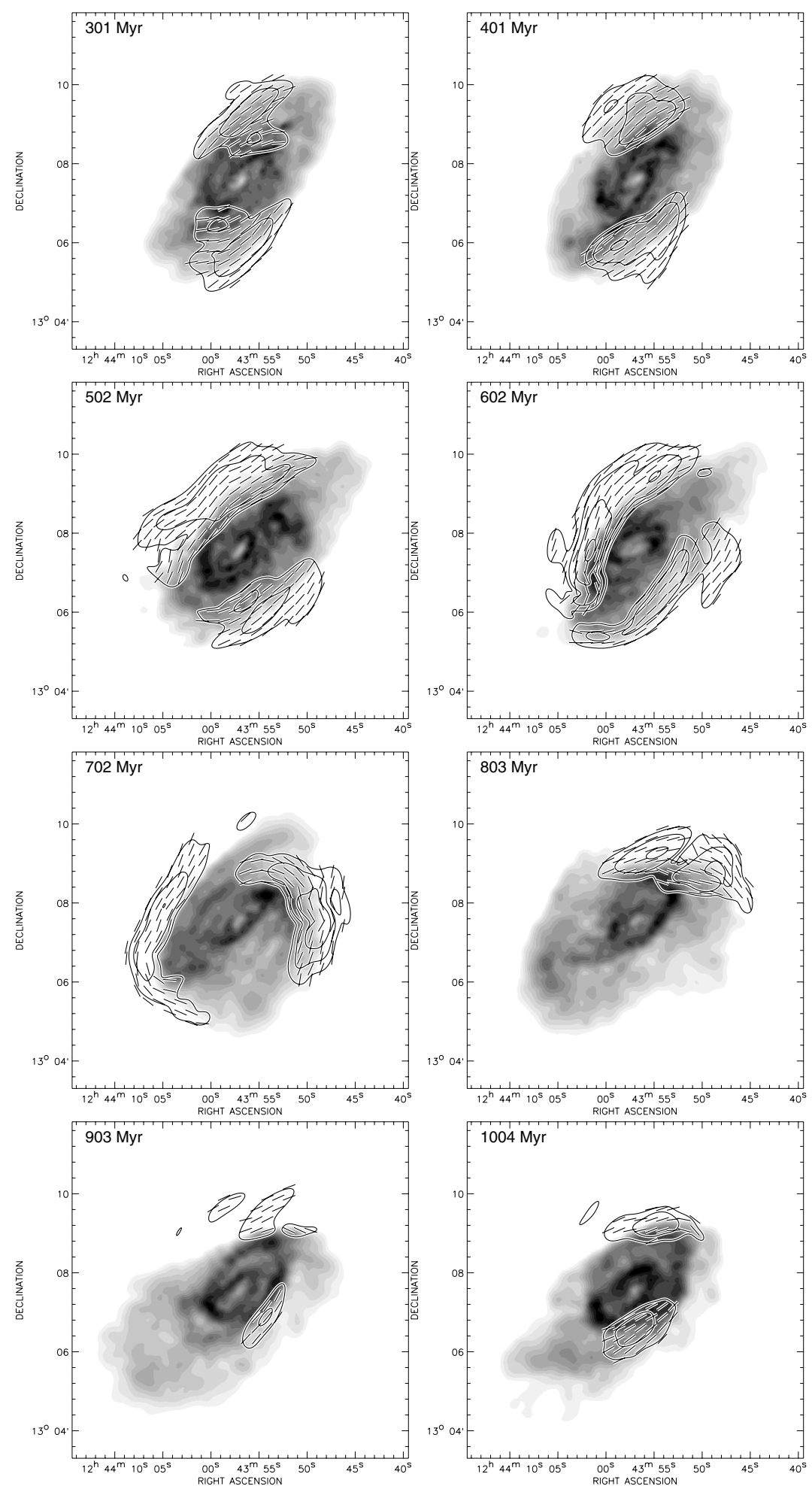

Fig. 3. Evolution of the polarized intensity at chosen time steps for the model only with gravitational interaction (model GR). Contours: polarized intensity in logarithmic scale. The polarized intensity vectors are superimposed onto the gas density distribution in logarithmic scale (grey plot). The contours are plotted at levels of $(1,3,10,30,100,300)$ (in arbitrary units) in all plots.

in consequence, the maxima of polarized intensity follow almost perfectly gas compression regions for $-50 \mathrm{Myr} \leq t \leq 200 \mathrm{My}$. Shortly before and after the maximum phase of the stripping event $(t=-40 \mathrm{Myr}$ and $t=80 \mathrm{Myr})$, we observe a bright maximum of polarized radio-continuum emission in the northeast of the galaxy center, at the location where the galaxy's ISM is compressed by ram pressure. At $t=200 \mathrm{Myr}$ ram pressure and compression decrease and rotation takes the magnetic field along with it. Since the galaxy rotates counter-clockwise, the result is an additional maximum of polarized emission in the south of the galaxy center. Rotation then makes the distribution of polarized emission approximately circular $(t=320 \mathrm{Myr})$. At $t>500 \mathrm{Myr}$ the stripped gas that was not accelerated to the escape velocity begins to fall back onto the galactic disk in the southeast of 

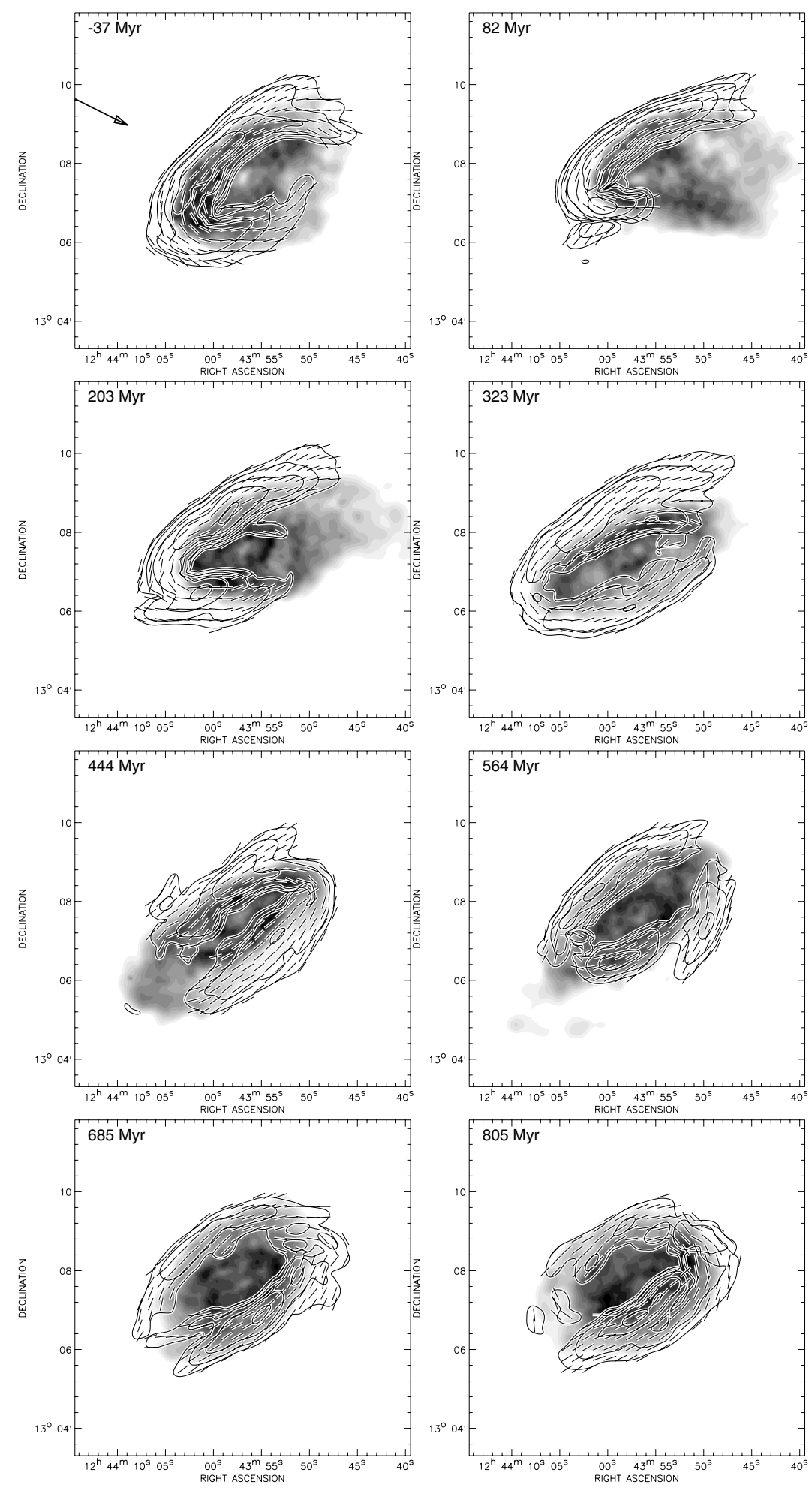

Fig. 4. Evolution of the polarized intensity at chosen time steps for the model with ram pressure event alone (model RPS). Contours: polarized intensity in logarithmic scale. The polarized intensity vectors are superimposed onto the gas density distribution in logarithmic scale (grey plot). The contours are plotted at levels of $(1,3,10,30,100,300)$ (in arbitrary units) in all plots. The arrow in the top left panel indicates the direction of the ram pressure wind, i.e. it is opposite to the galaxy's motion within the intracluster medium.

the galaxy center (re-accretion). This leads to shear motion that enhances the large-scale magnetic field and thus the polarized radio-continuum emission. Re-accretion of stripped gas then becomes stronger with time leading to a stronger maximum of polarized emission in the south of the galaxy center (see also Fig. 9 of Otmianowska-Mazur \& Vollmer 2003). Differences between the polarized emission and the gas distributions are due to (i) the longer timescale of turbulent diffusion compared to the viscous timescale and (ii) orientation (integration along the lineof-sight) and beam depolarization effects. The final stage of the magnetic field evolution shows an approximately circular distribution of polarized radio-continuum emission with a maximum 
in the north and another maximum to the south of the galaxy center. The southern maximum is brighter than the northern one.

\subsection{Gravitational interaction and ram pressure (model GRPS)}

Only when a weak, constant ram pressure is added to the gravitational interaction between NGC 4654 and NGC 4639 are the observed HI distribution and velocity field of NGC 4654 reproduced (Vollmer 2003). When a small constant ram pressure is added, the magnetic field and thus the evolution of the polarized radio-continuum emission changes considerably (Fig. 5). Again, it is possible to compare Fig. 5 directly to the snapshots of Fig. 14 of Vollmer (2003). The polarized intensity maps already show a clear asymmetry at early time steps because of gas compression due to ram pressure. Already at $t=300 \mathrm{Myr}$ the polarized intensity maximum that corresponds to the southern maximum of Fig. 3 is weaker and displaced to the northwest. In addition, the polarized intensity maximum that corresponds to the northern maximum of Fig. 3 is brighter due to gas compression and also displaced to the northwest.

Around the timestep of closest approach between the two galaxies $(t=460 \mathrm{Myr})$, tidal forces exceed those due to ram pressure. Therefore, the distribution of the polarized radiocontinuum emission for $450 \mathrm{Myr}<t<600 \mathrm{Myr}$ is close to that of model GR (Fig. 3). At later time steps, gas compression due to ram pressure in the northwest and gas re-accretion in the southeast dominate the gas dynamics. This leads to a pronounced maximum of polarized emission in the northwest and an elongated ridge of polarized emission in the south of the galaxy center where the large low column-density HI tail originates (see Phookun \& Mundy 1995).

\subsection{Total magnetic energy evolution}

Figure 6 shows the evolution of the total magnetic energy integrated over the whole cube normalized to its initial value for our three numerical models: GR (tidal interaction only), RPS (the ram pressure effect alone) and GRPS (tidal interaction and constant ram pressure). The magnetic energy is calculated in a cylinder of $40 \mathrm{kpc}$ diameter and $5 \mathrm{kpc}$ height centered on the galaxy center.

To investigate the impact of the initial conditions of the magnetic field on the evolution of the total magnetic energy, we used 3 different initial magnetic field configurations:

(i) a "normal" disk: the magnetic field is constant up to a radius of $20 \mathrm{kpc}$, decreases with a Gaussian profile for $20 \mathrm{kpc}<$ $R<30 \mathrm{kpc}$ with a half width of $500 \mathrm{pc}$, and has a cutoff at $30 \mathrm{kpc}$. In the vertical direction the initial magnetic field strength is Gaussian function with a length scale of $500 \mathrm{pc}$ and a cutoff at $1 \mathrm{kpc}$;

(ii) a "thin" disk: the magnetic field is constant up to a radius of $15 \mathrm{kpc}$, decreases with a Gaussian profile for $15 \mathrm{kpc}<R<$ $20 \mathrm{kpc}$ with a half width of $500 \mathrm{pc}$, and has a cutoff at $20 \mathrm{kpc}$. In the vertical direction the initial magnetic field strength is Gaussian function with a length scale of $300 \mathrm{pc}$ and a cutoff at $600 \mathrm{pc}$;

(iii) a "thick" disk: the magnetic field is constant up to a radius of $25 \mathrm{kpc}$, decreases with a Gaussian profile for $25 \mathrm{kpc}<R<$ $40 \mathrm{kpc}$ with a half width of $500 \mathrm{pc}$, and has a cutoff at $40 \mathrm{kpc}$. In the vertical direction the initial magnetic field strength is a Gaussian function with a length scale of $700 \mathrm{pc}$ and a cutoff at $1.5 \mathrm{kpc}$.
We observe that the evolution of the total magnetic energy of the "normal" and "thin" disk initial conditions is very close. For $t<600$ Myr the total magnetic energy of the "thick" disk model is systematically lower than that of the other models; i.e. the total magnetic energy grows more slowly than for the other two initial conditions. The reason is that the magnetic field amplification mainly takes place in the inner part of the galaxy's disk. Therefore, the ratio between the magnetic energy of the amplified field and that of the initial field is smaller for a larger "thick" disk than the same ratio for a smaller "thin" or "normal" disk. At $t>700 \mathrm{Myr}$, there is no significant difference between the total energies for the simulations with different initial conditions. We conclude that the evolution of the total magnetic energy depends only marginally on the initial configuration of the magnetic field.

The total magnetic energy initially grows in all three models (GR, RPS, GRPS). This effect can be explained by the magnetic field amplification due to gradients in the velocity field in early evolutionary stages. The initial field growth saturates at the time step of $t \sim 450 \mathrm{Myr}$ for models with a gravitational interaction (models GR and GRPS) and at $t \sim 500 \mathrm{Myr}$ for model with ram pressure alone (model RPS). These time steps correspond to the closest encounter with NGC $4639(t=460 \mathrm{Myr})$ and to the timestep of maximum ram pressure ( $t=500 \mathrm{Myr}$ ). For $t>500$ Myr the tidal interaction pulls gas out of the galaxy, taking the magnetic field with it beyond the limits of our computation box. Therefore, the total magnetic field energy decreases in models GR and GRPS. After the phase of strong interaction $(t \sim 600 \mathrm{Myr})$, a part of the displaced gas falls back onto the galactic disk (phase of re-accretion, see Otmianowska-Mazur \& Vollmer 2003) and its radial flows and shears connected to differential rotation of the galactic disk trigger magnetic field amplification very efficiently. As a result the magnetic energy of models GR and GRPS increases again at $t=600 \mathrm{Myr}$. At $t>700$ Myr constant ram pressure modifies the gas dynamics creating shear and a compression region, which leads to a further enhancement of the total magnetic energy, which then saturates at $t=750 \mathrm{Myr}$.

Surprisingly, the enhancement of the total magnetic energy due to strong ram pressure compression (model RPS) is small $(\sim 10 \%)$. The characteristic timescale of the ram pressure stripping event (model RPS) is smaller than that of the gravitational interaction and re-accretion is stronger for model RPS. The subsequent shear motion makes the large-scale magnetic field increase at $t>600 \mathrm{Myr}$. With the diminishing of re-accretion and thus shear motions the magnetic energy decreases again at $t>800 \mathrm{Myr}$ and stays approximately constant for $t>1000 \mathrm{Myr}$.

In all models we observe a significant growth in total magnetic energy at early interaction stages. Only for the model that only includes ram pressure (model RPS), the total magnetic energy at $\Delta t=t-t_{0}>300$ (where $t_{0}$ is the time of strongest interaction) is significantly higher (a factor of two) than the initial value (see Otmianowska-Mazur \& Vollmer 2003). The stripping of the large scale magnetic field, together with the ISM, might represent a way to amplify the intracluster magnetic field. The observations of ICM show (see e.g. Widrow 2002, and references therein) that such fields exist widely in clusters.

We thus obtain a time-dependent global magnetic field amplification in all our models without any explicit magnetic dynamo action (dynamo coefficient $\alpha=0$ ). The amplification was found to work very efficiently in the re-accretion phase - when the gas initially pulled out of the galaxy by an external interaction falls back due to the gravitational attraction by the host galaxy (see Otmianowska-Mazur \& Vollmer 2003). 

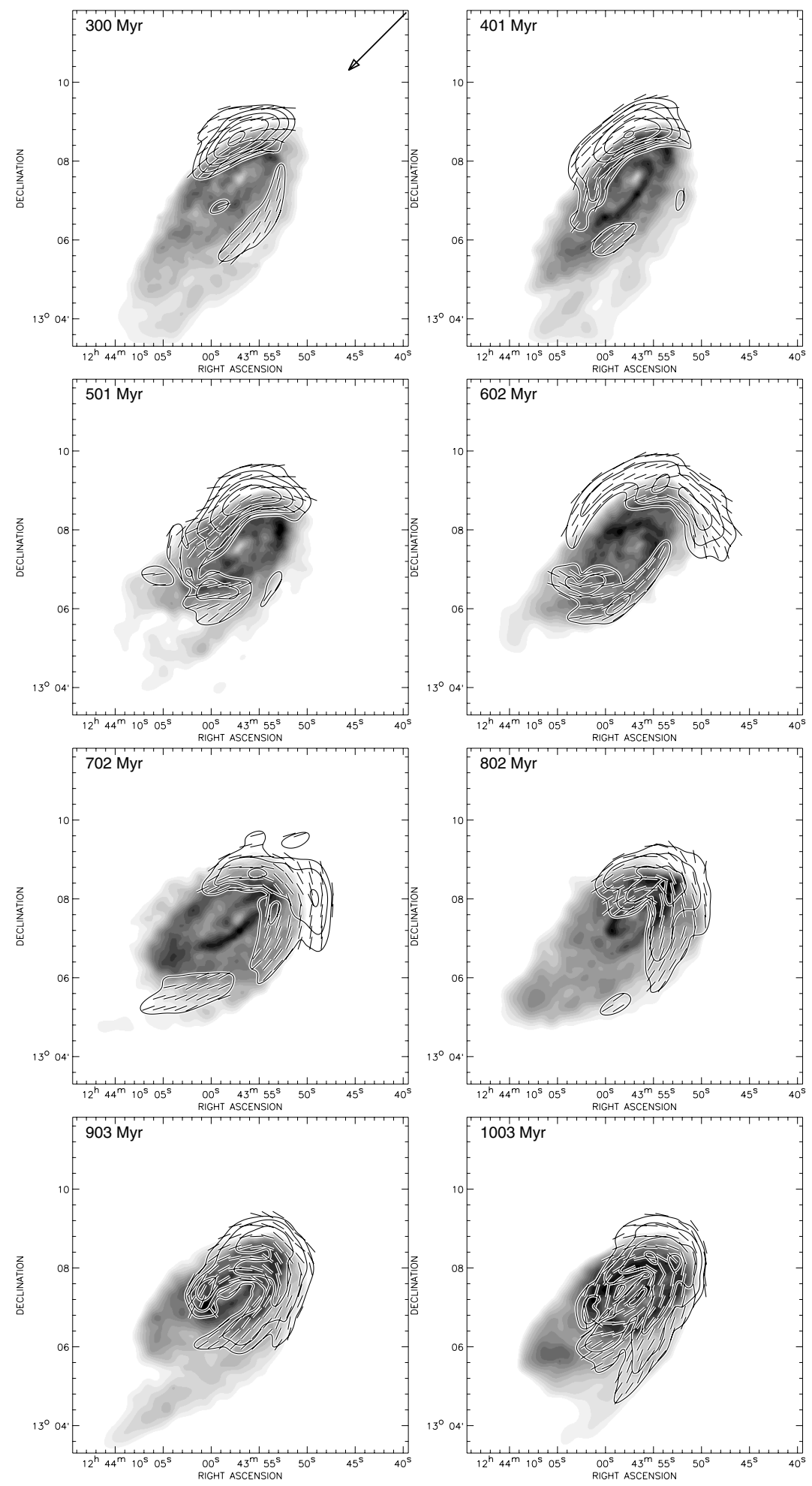

Fig. 5. Evolution of the polarized intensity at chosen time steps for the model with gravitational interaction and the constant ram pressure (model GRPS). Contours: polarized intensity in logarithmic scale. The polarized intensity vectors are superimposed onto the gas density distribution in logarithmic scale (grey plot). The contours are plotted at levels of $(1,3,10,30,100,300)$ (in arbitrary units) in all plots. The arrow in the top left panel indicates the direction of the ram pressure wind; i.e. it is opposite to the galaxy's motion within the intracluster medium.

\section{Models versus observations}

We first compare our models to the low-resolution Effelsberg data. This comparison can already distinguish between the different models. The high-resolution VLA data confirm the choice of the "best fit" model.

\subsection{The choice of the model polarized emission threshold}

As stated in Sect. 3.3 we can only compare the model to the observed polarized radio-continuum emission. The model polarized emission is calculated everywhere in the galaxy according to Eq. (9). For a proper comparison we need to know which level of the model polarized emission corresponds to the observed 

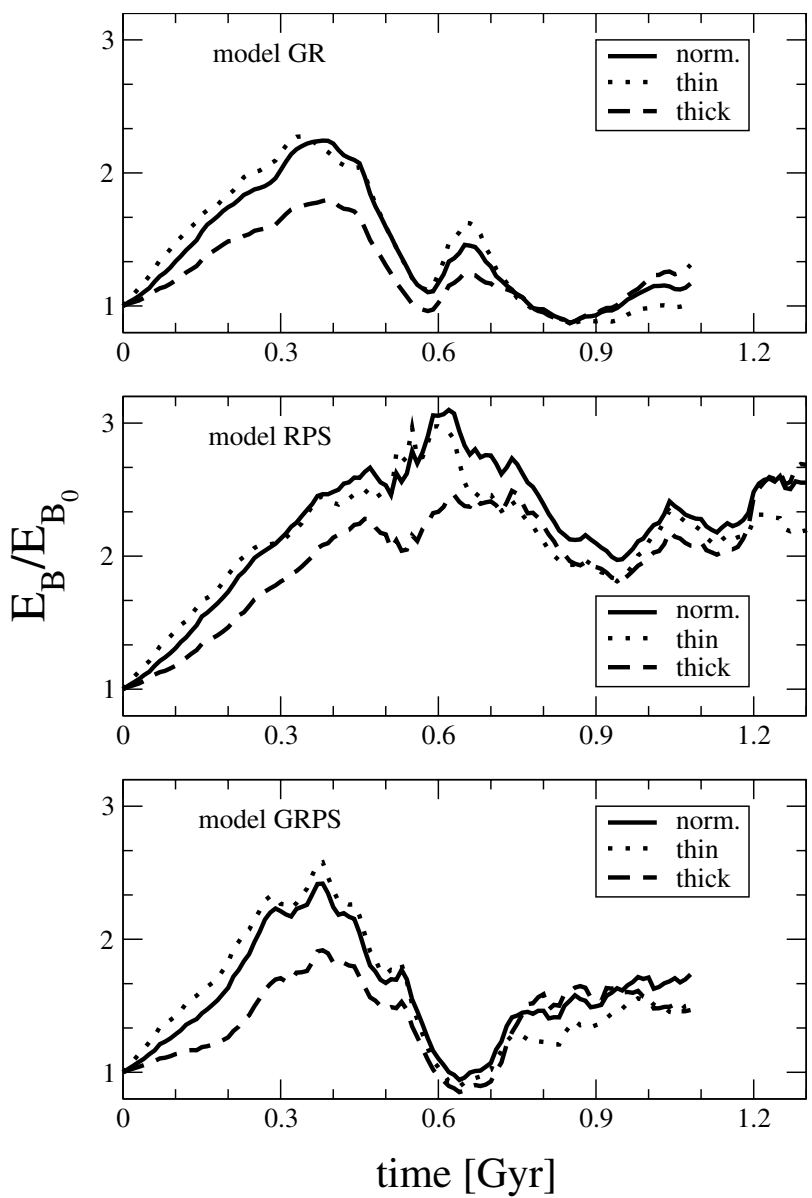

Fig. 6. Total magnetic energy evolution in our three models - ram pressure alone (model RPS), only gravitational interaction (model GR), and gravitational interaction with constant ram pressure (model GRPS).

rms noise level. Since we do not attempt to estimate the absolute synchrotron emissivity $\epsilon_{I}$, because of our lack of knowledge about the absolute magnetic field strength and relativistic electron density, we decided to use a fixed fraction of the maximum of the model polarized emission distribution as a model threshold (Figs. 8, 9). To give an impression of the polarized radiocontinuum distribution at lower flux densities, we show in Fig. 7 the last snapshots of Figs. 3-5 with one deeper contour level at 0.3 times the lowest contour of the corresponding snapshots. For the GR model (upper panel), the two maxima of Fig. 3 are connected by a ridge of faint, polarized emission. In addition, a faint emission region appears outside the main gas disk in the north, which is due to very low surface density gas (see Vollmer 2003). The faint component of the polarized emission distribution of the RPS model (middle panel) forms a ring structure. Moreover, it extends a little bit farther out than the 3 times stronger emission. This is also the case for the faint emission of the GRPS model (lower panel).

As a conclusion, the new polarized emission feature is due to very low surface-density gas in the case of the GR model. The lowest contours of the RPS and GRPS models (Fig. 7) (i) do not show any new feature; (ii) are only a little more extended than the former lowest contours; and (iii) are a natural extension of the higher contour levels. Our method is thus not very sensitive to the choice of the model threshold. The same is true for the convolved data.
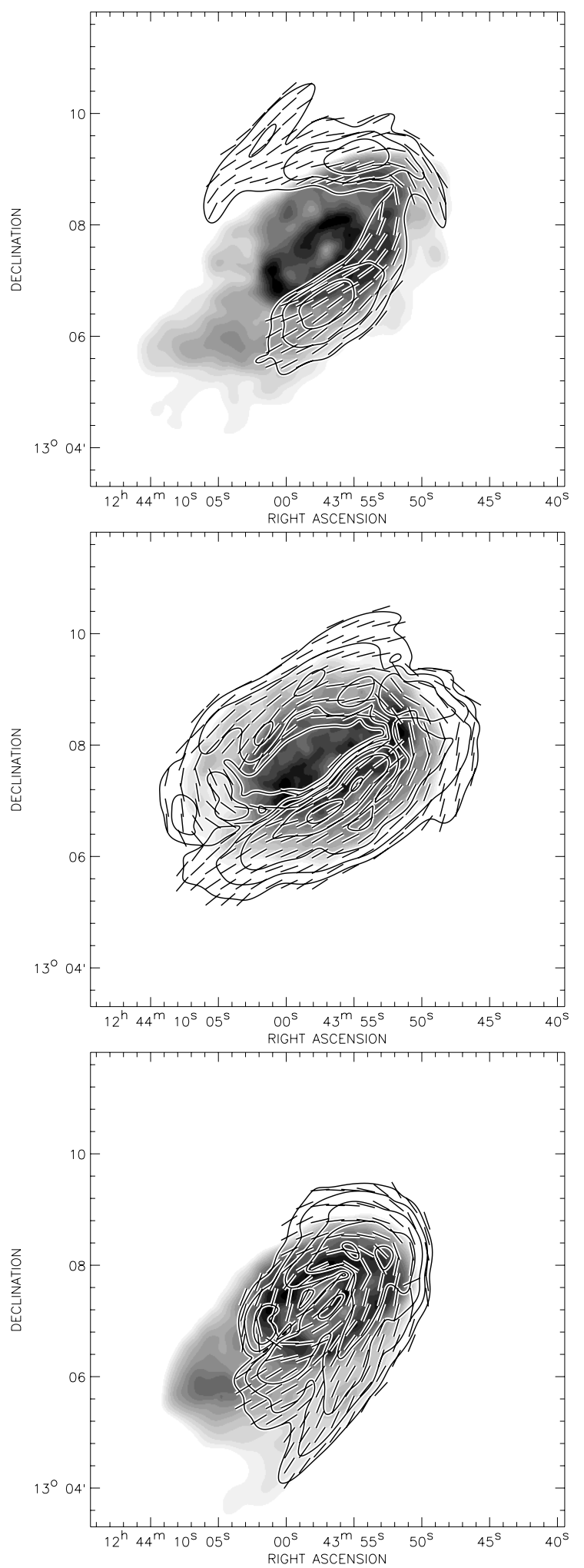

Fig. 7. Model polarized radio-continuum emission. Upper panel: GR, middle panel: RPS, lower panel: GRPS model. The contour levels are the same as in Figs. 3-5, but the first contour is 0.3 times that of these figures.

\subsection{The Effelsberg data}

To compare our simulations to our Effelsberg observations (Fig. 1), we convolved the final model snapshots to the Effelsberg resolution of $80^{\prime \prime} H P B W$ at $8.35 \mathrm{GHz}$. These 

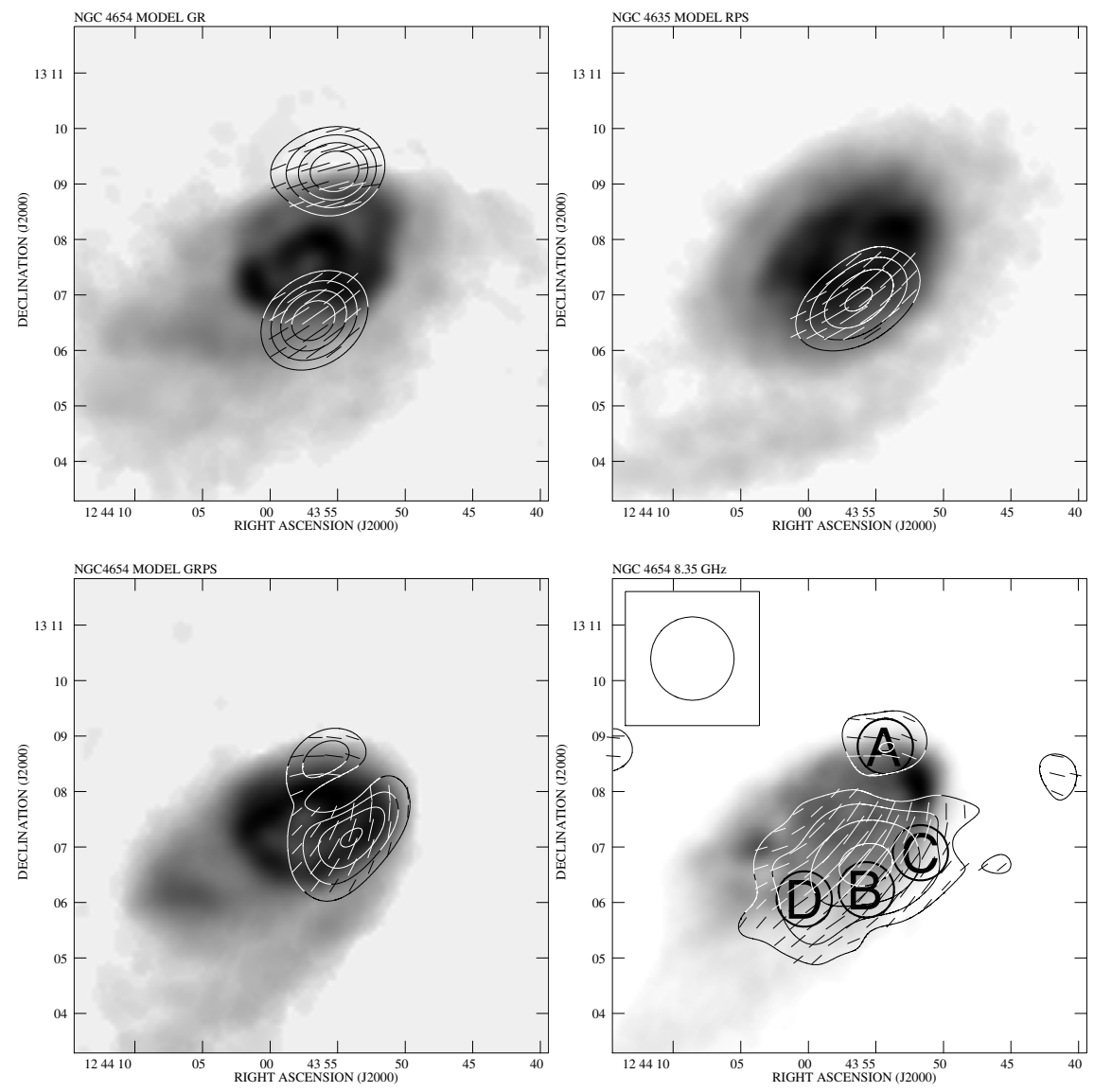

Fig. 8. Models versus observations for the Effelsberg data. Contours: polarized intensity. Grey scale: gas distribution. The magnetic field vectors are shown as lines. Top left: model with a gravitational interaction alone (GR). Contour levels are $(6,8,10,12) \times 2.5$ (in arbitrary units). Top right: model with ram pressure stripping alone (RPS). Contour levels are $(6,8$, $10,12) \times 15$ (in arbitrary units). Bottom left: model with a tidal interaction and a small constant ram pressure (GRPS). Contour levels are $(6,8,10,12) \times 10$ (in arbitrary units). Bottom right: greyscale: HI gas distribution (Phookun \& Mundy 1995); contours: Effelsberg $8.35 \mathrm{GHz}$ observations. Contour levels are $(3,5,8,12) \times 40 \mu \mathrm{Jy} /$ beam . convolved snapshots can be seen in Fig. 8 together with our Effelsberg observations. All three numerical models lead to two maxima of polarized intensity, located north and south of the galaxy center. The second maximum of model RPS cannot be seen, because it is too weak.

There are significant differences in the distribution and strength of the polarized intensity maxima among our models. The model only with gravitational interaction (model GR, Fig. 8, top left) shows two maxima of almost equal intensity. The model with ram pressure alone (model RPS, Fig. 8, top right) has only one bright maximum in the south and a very faint maximum in the north of the galaxy center that is 6.5 times weaker than the southern maximum. The simulation including a tidal interaction and a small constant ram pressure (model GRPS, Fig. 8, bottom left) shows two maxima with the northern one 1.3 times weaker than the southern one. While the locations of the northern maximum are the same for all three models, the location of the southern maximum is most eastward in model GR, westward in model GRPS, and in between in model RPS.

\subsection{The VLA data}

To compare the simulations to our VLA observations (Fig. 2), we convolved the final model snapshots to the VLA resolution of $20^{\prime \prime} H P B W$ at $4.85 \mathrm{GHz}$. These convolved snapshots can be seen in Fig. 9 together with our VLA observations. Since the VLA image has a smaller r.m.s. noise level than the Effelsberg image, we plot deeper contours in the model polarized radio continuum emission maps. There is almost no difference between the low and high resolution maps of the GR model, i.e. there are two distinct maxima in the north and the south of the galaxy. The low-resolution map of the RPS model only shows the main ridge of polarized emission, whereas the high resolution map shows an additional faint ring-like structure. The difference between the high and low resolution model polarized emission maps is most significant for the GRPS model where the deep high-resolution map reveals a complex structure. The main ridge of polarized emission is located in the west and southwest of the galaxy center. In addition, a distinct maximum can be found in the north west at the edge of the gas disk. Polarized emission can be found within the entire southwestern quadrant of the galactic disk. The southern part of the polarized emission distribution splits into two arms in the southeast direction. One arm is located within the inner gas disk, the other arm extends in the direction of the extended HI tail. The inner arm is due to the tidal interaction, whereas the outer arm is mainly caused by ram pressure.

\section{Discussion}

The GR model produces neither the location of the southern maximum nor the intensity ratio between the two maxima. While the location of the southern maximum is reproduced best by model RPS, the intensity ratio between the two maxima is much too large. Only the model including a gravitational interaction with NGC 4639 and a constant ram pressure (model GRPS) reproduces our Effelsberg observations in a qualitatively satisfactory way. Both maxima are placed at about the correct positions, and the southern maximum is brighter than the northern one, although the intensity ratio is still too small (about 1.3 in the model compared to 2.4 in our observations).

As a further step, we compare the magnetic field orientation in our models with that of our observations. We calculated the mean values of the pitch angles of the magnetic vectors in four chosen regions of the galactic disk - one at the northern 
Table 1. Mean magnetic pitch-angle values calculated in four chosen regions of the real galaxy and our three models' disks (see Fig. 8) - ram pressure alone (Model RPS), gravitational interaction (Model GR), and gravitational interaction with constant ram pressure (model GRPS).

\begin{tabular}{cccrrrr}
\hline \hline & $\begin{array}{c}\alpha_{2000} \\
{[\mathrm{~h} \mathrm{~m} \mathrm{~s}]}\end{array}$ & $\begin{array}{c}\delta_{2000} \\
{\left[{ }^{\circ}{ }^{\prime \prime}\right]}\end{array}$ & $\begin{array}{r}\text { NGC } \\
4654\end{array}$ & GR & RPS & GRPS \\
\hline $\mathrm{A}$ & 124354.3 & 130848.8 & -4 & 19 & 3 & -9 \\
$\mathrm{~B}$ & 124355.7 & 130613.8 & 19 & 7 & 3 & 27 \\
$\mathrm{C}$ & 124351.7 & 130653.8 & -18 & -34 & -33 & -7 \\
$\mathrm{D}$ & 124400.3 & 130603.8 & 46 & 41 & 36 & 44 \\
\hline
\end{tabular}

polarized intensity maximum, and three others in the region of the southern polarized intensity maximum: at the location of the maximum and on both sides (at a distance of one beam size, see Fig. 8). These measurements are shown in Table 1 . The models with a gravitational interaction alone (GR) and with ram pressure alone (RPS) yield pitch angles too small that are on the southern maximum. On the other hand, the pitch angle of the northern maximum of model GR is too large with respect to the observed value. The best agreement between the model and observed pitch angles for all positions is found for model GRPS.

The two prominent maxima of the high-resolution model's polarized emission map of the GR model are not present in the VLA observations. At first glance, the overall polarized emission distribution of the RPS model seems to fit observations quite well. However, the faint northern maximum is not observed, there is no continuum emission in the inner gas disk, and the southern maximum is about twice as strong as observed. Only the GRPS model's polarized emission distribution shows the key features of the VLA observations: (i) a distinct maximum can be found in the northwest at the edge of the gas disk. This maximum is due to gas compression by ram pressure; (ii) polarized emission can be found within the entire southwestern quadrant of the galactic disk; (iii) there is a region of strong polarized emission in the southwest of the galaxy. However, we do not observe the bifurcation of the polarized emission ridge to the southeast. Especially the arm pointing toward the extended HI tail is missing in the observations. This might be due to a difference between the model and real distribution of relativistic electrons. Despite this disagreement, we feel confident that the GRPS model reproduces our VLA observations best and thus confirms our findings based only on the Effelsberg data.

We conclude that GRPS model not only successfully reproduces the HI distribution and velocity field, but also successfully reproduces the observed polarized radio-continuum emission distribution. Observations of polarized emission can serve as an additional diagnostic tool for galactic interactions (see Otmianowska-Mazur \& Vollmer 2003). It can add the information on gas flows perpendicular to the line of sight, adding a new dimension to radial velocity studies (see also Urbanik 2005).

In the case of NGC 4654 we identified the northwestern polarized emission maximum as due to ram pressure. As in NGC 4522 (Vollmer et al. 2006), we can detect the compression of the interstellar medium directly in polarized radio-continuum emission. This independently confirms the claim of Phookun \& Mundy (1995) that the linear HI rotation curve in the northwestern part of the galaxy is due to compression and the same conclusion of Vollmer (2003) which was based on the comparison between deep HI data and a dynamical model. In the case of NGC 4522 (Vollmer et al. 2006), it was not even possible to detect the compression region in the HI velocity field. A part of the southern polarized radio continuum emission ridge is due to the tidal interaction, which is also responsible for the one-armed spiral. Another part is due to shear motions induced by the action of ram pressure. It is very difficult to determine regions of shear motions only on the basis of a radial velocity field. Since the polarized radio continuum emission is sensitive to the $3 \mathrm{D}$ velocity field, one can gain important information about transversal motions. By definition, this information is not contained in a radial velocity field. Thus, polarized radio continuum emission gives us important information about shear and compression regions that may not be accessible via velocity fields.

Our present study corroborates the results of Vollmer (2003), that NGC 4654 had a recent close and rapid tidal interaction with NGC 4639. Cepheid measurements (Gibson et al. 2000; Freedman et al. 2001) place NGC 4639 several Mpc behind the Virgo cluster center (M 87, see Sect. 1). However, H-band TullyFisher measurements (Gavazzi et al. 1999) yield a distance of NGC 4654 of 1-2 Mpc in front of the Virgo cluster center. In light of our new results, we suggest that the Tully-Fisher distance is underestimated and that the line-of-sight distance of NGC 4654 is close to that of NGC 4639.

Another result of our study is that a small amount of ram pressure is needed to reproduce the HI gas distribution, velocity field, and the distribution of the polarized radio-continuum emission. An intracluster medium (ICM) density of $\sim 10^{-4} \mathrm{~cm}^{-3}$ is needed for this ram pressure (see Sect. 1). If the ICM density distribution is spherical, the density at a distance of $5 \mathrm{Mpc}$ is more than a magnitude smaller. A density of $10^{-4} \mathrm{~cm}^{-3}$ is already reached at a distance of $1 \mathrm{Mpc}$ from the cluster center (Schindler et al. 1999). We therefore suggest that the ICM distribution of the Virgo cluster is not spherical but elongated in the line of sight with an axis ratio of about 5:1 as observed for the galaxy distribution (Gavazzi et al. 1999; Yasuda et al. 1997).

\section{Conclusions}

The Virgo spiral galaxy NGC 4654 was observed with the Effelsberg $100 \mathrm{~m}$ telescope at $8.35 \mathrm{GHz}$ and the VLA at $4.85 \mathrm{GHz}$ in polarization. We find an asymmetric distribution of the polarized intensity with a strong peak in the south of the galaxy. This shows that asymmetric polarized radio continuum distributions are typical of cluster spiral galaxies (Vollmer et al. 2004; Urbanik 2005).

The polarized radio continuum emission was used as an additional diagnostic tool for the interaction between NGC 4654 and its environment. To do so, we solved the induction equation for the dynamical model evolution of the interstellar medium of a cluster spiral galaxy and calculated the large-scale magnetic field in this way. With an assumed distribution of relativistic electrons, the distribution of polarized radio-continuum emission can be calculated and directly compared to our observations. The input velocity fields are those from the simulations of Vollmer (2003), who simulated three different interaction scenarios: (i) a gravitational interaction between NGC 4654 and NGC 4639; (ii) ram pressure stripping by the intracluster medium; and (iii) a gravitation interaction, along with a small constant ram pressure.

The direct comparison between our MHD simulations and the polarized radio-continuum observations shows that only the model with gravitation interaction and low, constant ram pressure can reproduce observations in a satisfactory way. We found that the low-resolution Effelsberg data are sufficient for distinguishing between the models. Thus, our study corroborates the results of Vollmer et al. (2004). This mixed interaction succeeds in reproducing the HI gas distribution and velocity field (Vollmer 2003) and the polarized radio-continuum distribution. 

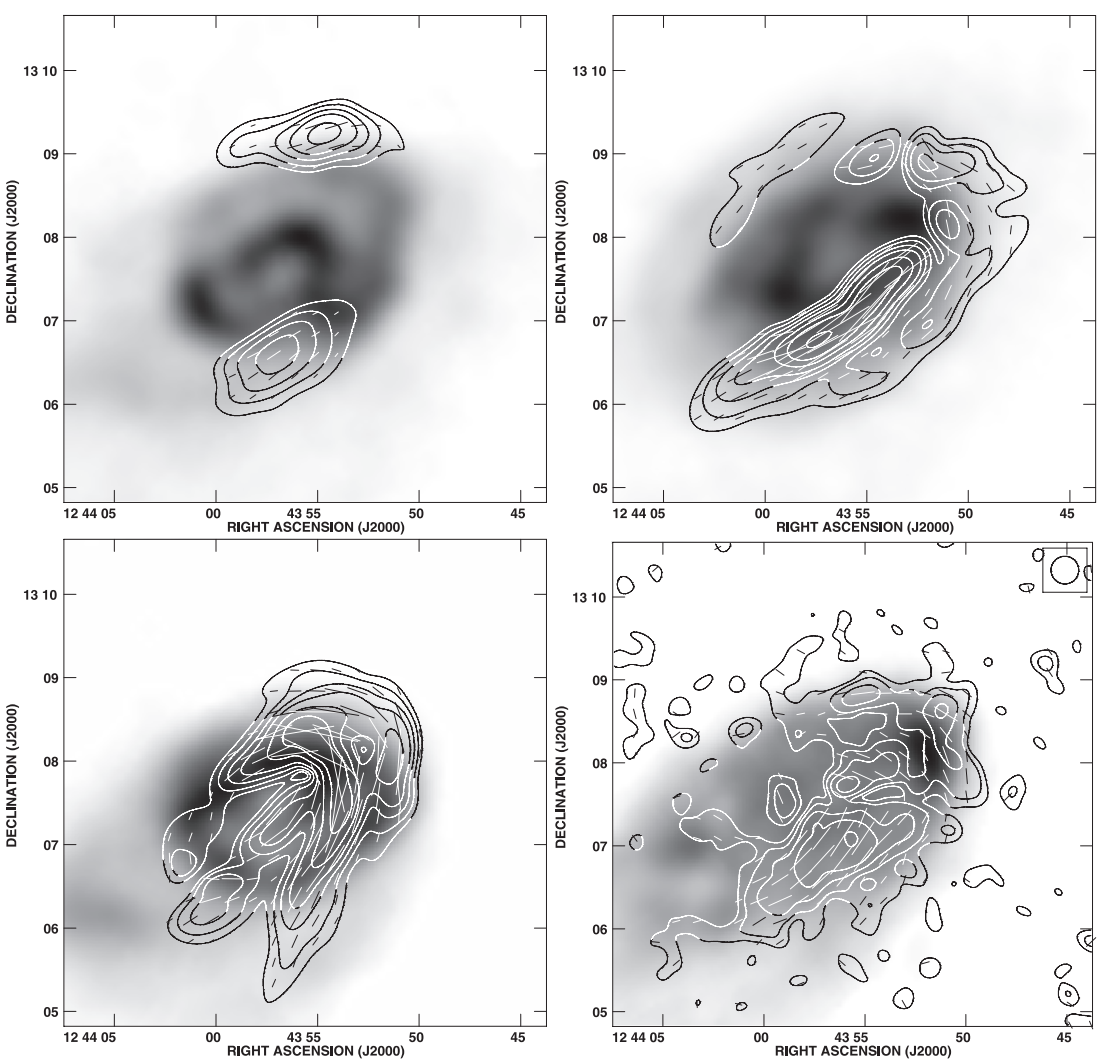

Fig. 9. Models versus observations for the VLA data. Contours: polarized intensity. Grey scale: gas distribution. The magnetic field vectors are shown as lines. Top left: model with a gravitational interaction alone (GR). Contour levels are $(3,5,8,12,20,30) \times 4.3$ (in arbitrary units). Top right: model with ram pressure stripping alone (RPS). Contour levels are $(3,5,8,12,20$, $30) \times 8$ (in arbitrary units). Bottom left: model with a tidal interaction and a small constant ram pressure (GRPS). Contour levels are $(3,5,8$, $12,20,30) \times 8$ (in arbitrary units). Bottom right: greyscale: Hi gas distribution (Phookun \& Mundy 1995); contours: VLA $4.85 \mathrm{GHz}$ observations. Contour levels are $(3,5,8,12,20$, 30) $\times 8 \mu \mathrm{Jy} /$ beam.
Since Cepheid measurements place NGC $4639 \sim 5$ Mpc behind the center of the Virgo cluster, we suggest that the distribution of the Virgo intracluster medium is highly elongated thereby providing the necessary ram pressure. Thus, polarized radio-continuum emission, in combination with detailed MHD modeling, provides an important additional diagnostic tool for studying interactions between a galaxy and its cluster environment. This tool is complementary to deep HI observations.

Acknowledgements. This work was supported by the Polish-French (ASTROLEA-PF) cooperation program, and by the Polish Ministry of Sciences grant PB 378/P03/28/2005.

\section{References}

Baars, J. W. M., Genzel, R., Pauliny-Toth, I. I. K., \& Witzel, A. 1977, A\&A, 61, 99

Beck, R., Brandenburg, A., Moss, D., Shukurov, A., \& Sokoloff, D. 1996, ARA\&A, 34, 155

Cayatte, V., Kotanyi, C., Balkowski, C., \& van Gorkom, J. H. 1994, AJ, 107, 1003

Emerson, D. T., \& Gräve, R. 1988, A\&A, 190, 353

Freedman, W. L., Madore, B. F., Gibson, B. K., et al. 2001, ApJ, 533, 47
Gavazzi, G., Boselli, A., Scodeggio, M., Pierini, D., \& Belsole, E. 1999, MNRAS, 304, 595

Gibson, B. K., Stetson, P. B., Freedman, W. L., et al. 2000, ApJ, 529, 723

Haslam, C. G. T. 1974, A\&AS, 15, 333

Isaaks, E. H., \& Srivastava, R. 1989, An Introduction to Applied Geostatistics (Oxford University Press), Chap. 12

Knapik, J., Soida, M., Urbanik, M., Dettmar, R.-J., \& Beck, R. 2000, A\&A, 362, 910

Koopmann, R. A., Kenney, J. D. P., \& Young, J. 2001, ApJS, 135, 125

Minchin, R. F., Davies, J. I., Disney, M. J., et al. 2005, AAS, 37, 1484

Otmianowska-Mazur, K., \& Vollmer, B. 2003, A\&A, 402, 879

Otmianowska-Mazur, K., Chyży, K., \& Soida, M. 2000, A\&A, 359, 29

Otmianowska-Mazur, K., Elstner, D., Soida, M., \& Urbanik, M. 2002, A\&A, 384,48

Phookun, B., \& Mundy, L. G. 1995, ApJ, 453, 154

Schindler, S., Binggeli, B., \& Böhringer, H. 1999, A\&A, 343, 420

Stone, J. M., \& Norman, M. L. 1992a, ApJS, 80, 791

Stone, J. M., \& Norman, M. L. 1992b, ApJS, 80, 791

Urbanik, M. 2005, in Proc. of Magnetized Plasma in Galaxy Evolution, Jagiellonian University, Kraków, 2005, ed. K. T. Chyży, K. Otmianowska-Mazur, M. Soida, \& R.-J. Dettmar

Vollmer, B. 2003, A\&A, 398, 525

Vollmer, B., Beck, R., Kenney, J. D. P., \& van Gorkom, J. H. 2004, AJ, 127, 3375

Vollmer, B., Soida, M., Otmianowska-Mazur, K., et al. 2006, A\&A, 453, 883

Warmels, R. H. 1988, A\&AS, 72, 57

Widrow, L. M. 2002, Rev. Mod. Phys., 74, 775

Yasuda, N., Fukugita, M., \& Okamura, S. 1997, ApJ, 108, 417 